\title{
Conditional Steady-State Bounds for a Subset of States in Markov Chains
}

\author{
Tuğrul Dayar \\ Department of Computer \\ Engineering \\ Bilkent University \\ TR-06800 Bilkent, Ankara, \\ Turkey \\ tugrul@cs.bilkent.edu.tr
}

\author{
Nihal Pekergin \\ PRiSM \\ Université de \\ Versailles-St.Quentin \\ 45 av. des Etats Unis \\ 78035 Versailles, France \\ and \\ Centre Marin Mersenne \\ Université Paris 1 \\ 90 rue Tolbiac \\ 75013 Paris, France \\ nih@prism.uvsq.fr
}

\author{
Sana Younès \\ PRiSM \\ Université de \\ Versailles-St.Quentin \\ 45 av. des Etats Unis \\ 78035 Versailles, France \\ sayo@prism.uvsq.fr
}

\begin{abstract}
The problem of computing bounds on the conditional steadystate probability vector of a subset of states in finite, ergodic discrete-time Markov chains (DTMCs) is considered. An improved algorithm utilizing the strong stochastic (st-)order is given. On standard benchmarks from the literature and other examples, it is shown that the proposed algorithm performs better than the existing one in the strong stochastic sense. Furthermore, in certain cases the conditional steadystate probability vector of the subset under consideration can be obtained exactly.
\end{abstract}

\section{Categories and Subject Descriptors}

C.4 [Performance of Systems]: Modeling techniques; G.3 [Probability and Statistics]: Markov processes; G.1.3 [Numerical Analysis]: Numerical Linear Algebra-Sparse, structured, and very large systems (direct and iterative methods)

\section{General Terms}

Algorithms, Performance, Theory

\section{Keywords}

Markov chains, conditional steady-state vector, stochastic comparison, strong stochastic order, bounding

${ }^{*}$ The work of the first author is supported by the Turkish Academy of Sciences grant TÜBA-GEBIP and that of the last two authors is supported by EURO-NGI network of excellence from the 6th PCRD.

Permission to make digital or hard copies of all or part of this work for personal or classroom use is granted without fee provided that copies are not made or distributed for profit or commercial advantage and that copies bear this notice and the full citation on the first page. To copy otherwise, to republish, to post on servers or to redistribute to lists, requires prior specific permission and/or a fee.

SMCTools'06, October 10, 2006, Pisa, Italy

Copyright 2006 ACM 1-59593-506-1/06/10...\$5.00

\section{INTRODUCTION}

Let $P$ denote the transition probability matrix of an irreducible discrete-time Markov chain (DTMC) [14] defined on the finite state space $\mathcal{S}$ with $n$ states and the block partitioning

$$
P=\left[\begin{array}{ll}
P_{\mathcal{A}, \mathcal{A}} & P_{\mathcal{A}, \mathcal{B}} \\
P_{\mathcal{B}, \mathcal{A}} & P_{\mathcal{B}, \mathcal{B}}
\end{array}\right] \begin{gathered}
n_{\mathcal{A}} \\
n_{\mathcal{B}}
\end{gathered},
$$

where $\mathcal{A} \cup \mathcal{B}=\mathcal{S}, \mathcal{A} \cap \mathcal{B}=\emptyset$, and $n_{\mathcal{A}}$ and $n_{\mathcal{B}}$ are respectively the number of states in subsets $\mathcal{A}$ and $\mathcal{B}$, implying $n=$ $n_{\mathcal{A}}+n_{\mathcal{B}}$. Note that for given $\mathcal{A} \subset \mathcal{S}, P$ can always be symmetrically permuted to the block form in (1). Here $P_{\mathcal{A}, \mathcal{A}}$ is the square submatrix of order $n_{\mathcal{A}}$ obtained from $P$ by deleting the rows and columns associated with states in $\mathcal{B}$. Being an irreducible DTMC, $P$ satisfies $P \geq 0$ and $P e=e$, where $e$ is column vector of ones with appropriate length. Furtermore, $P_{\mathcal{A}, \mathcal{A}}$ is substochastic, meaning $P_{\mathcal{A}, \mathcal{A}} \geq 0$ and $P_{\mathcal{A}, \mathcal{A}} e \leq e$, but $P_{\mathcal{A}, \mathcal{A}} e \neq e$.

The stochastic complement of $P_{\mathcal{A}, \mathcal{A}}$, denoted by $S_{\mathcal{A}}$, is the irreducible DTMC given by [8]

$$
\begin{aligned}
S_{\mathcal{A}} & =P_{\mathcal{A}, \mathcal{A}}+\underbrace{P_{\mathcal{A}, \mathcal{B}}\left(I-P_{\mathcal{B}, \mathcal{B}}\right)^{-1} P_{\mathcal{B}, \mathcal{A}}}_{H_{\mathcal{A}, \mathcal{A}}} \\
& =P_{\mathcal{A}, \mathcal{A}}+H_{\mathcal{A}, \mathcal{A}} .
\end{aligned}
$$

It is well known that $\left(I-P_{\mathcal{B}, \mathcal{B}}\right)$ is a nonsingular M-matrix [2], implying $\left(I-P_{\mathcal{B}, \mathcal{B}}\right)^{-1} \geq 0$. This, combined with $P_{\mathcal{A}, \mathcal{A}} \geq 0$ and $P_{\mathcal{B}, \mathcal{A}} \geq 0$, suggests $H_{\mathcal{A}, \mathcal{A}} \geq 0$. Observe that $H_{\mathcal{A}, \mathcal{A}} e=$ $e-P_{\mathcal{A}, \mathcal{A}} e$ since $S_{\mathcal{A}}$ is an irreducible DTMC. In particular, $S_{\mathcal{A}}$ is the sum of two terms, the first of which represents transitions within $\mathcal{A}$, and the second of which represents transitions from $\mathcal{A}$ to $\mathcal{B}$, spending some nonnegative time in $\mathcal{B}$, and then returning to $\mathcal{A}$ (see equation (2)). The probability of moving directly from $a_{i} \in \mathcal{A}$ to $a_{j} \in \mathcal{A}$ is $P_{\mathcal{A}, \mathcal{A}}\left[a_{i}, a_{j}\right]$ and the probability of moving from $a_{i} \in \mathcal{A}$ to $a_{j} \in \mathcal{A}$ through states in $\mathcal{B}$ is $H_{\mathcal{A}, \mathcal{A}}\left[a_{i}, a_{j}\right]$. We remark that similar statements can be made regarding the stochastic complement of $P_{\mathcal{B}, \mathcal{B}}$. In summary, the stochastic complement associated with a subset of states in an irreducible DTMC is an irreducible DTMC representing the evolution of the original process restricted to the subset of states. For this reason, 
some texts refer to the stochastic complement as the censored $M C$ (see, for instance, [3]).

Now, let us further assume that $P$ is aperiodic (meaning it is ergodic since we already assumed it to be irreducible), implying the existence of a unique, positive steady-state probability distribution (row) vector $\pi=\left[\begin{array}{ll}\pi_{\mathcal{A}} & \pi_{\mathcal{B}}\end{array}\right]$ conformally partitioned with $P$ such that

$$
\begin{aligned}
\pi P & =\left[\begin{array}{ll}
\pi_{\mathcal{A}} & \pi_{\mathcal{B}}
\end{array}\right]\left[\begin{array}{ll}
P_{\mathcal{A}, \mathcal{A}} & P_{\mathcal{A}, \mathcal{B}} \\
P_{\mathcal{B}, \mathcal{A}} & P_{\mathcal{B}, \mathcal{B}}
\end{array}\right] \\
& =\left[\begin{array}{ll}
\pi_{\mathcal{A}} & \pi_{\mathcal{B}}
\end{array}\right]=\pi \text { with } \pi e=1 .
\end{aligned}
$$

Throughout the text we assume all probability vectors to be row vectors. Now, if $\pi_{S_{A}}$ denotes the steady-state probability distribution vector of $S_{\mathcal{A}}$ (that is, $\pi_{S_{\mathcal{A}}} S_{\mathcal{A}}=\pi_{S_{\mathcal{A}}}$ with $\left.\pi_{S_{\mathcal{A}}} e=1\right)$, then [8]

$$
\pi_{S_{\mathcal{A}}}=\pi_{\mathcal{A}} /\left(\pi_{\mathcal{A}} e\right) .
$$

In fact, the steady-state vector of the stochastic complement $S_{\mathcal{A}}$ represents the conditional steady-state probability of its states given that the DTMC is in subset $\mathcal{A}$.

In practical problems $P$ is large, and therefore it is expensive to form $S_{\mathcal{A}}$ unless $n_{\mathcal{B}} \ll n$ (see equations (1) and (2)) This simply follows from the fact that the computation of $S_{\mathcal{A}}$ requires factorizing the matrix $\left(I-P_{\mathcal{B}, \mathcal{B}}\right)$ of order $n_{\mathcal{B}}$ and performing $n_{\mathcal{A}}$ forward and backward substitutions, each using a different column of $P_{\mathcal{B}, \mathcal{A}}$ as the right-hand side, to obtain $\left(I-P_{\mathcal{B}, \mathcal{B}}\right)^{-1} P_{\mathcal{B}, \mathcal{A}}$. Since it is mostly $\pi_{S_{\mathcal{A}}}$ in equation (3) rather than $S_{\mathcal{A}}$ in equation (2) that is sought, an alternative approach would be to compute bounds on $\pi_{S_{\mathcal{A}}}$ without forming $S_{\mathcal{A}}$. It is this kind of approach that we consider in this paper. Such an approach is taken, for instance, at the second level of the two-level bounded aggregation method [5], which is based on polyhedra theory and geared towards nearly completely decomposable (NCD) MCs [14]. The theory essentially says that one can compute bounds on $\pi_{S_{\mathcal{A}}}$ by factorizing the matrix $\left(I-P_{\mathcal{A}, \mathcal{A}}\right)^{T}$ of order $n_{\mathcal{A}}$ and performing $n_{\mathcal{A}}$ forward and backward substitutions, each using a different column of $I$ as the right-hand side, under a normalization condition. The bounds obtained in this manner are known to provide the best bounds that can be attained by solely using the information available in $P_{\mathcal{A}, \mathcal{A}}$ and are especially tight for NCD MCs.

Here, we take a different view and consider the stochastic comparison approach to bound $\pi_{S_{\mathcal{A}}}$ in equation (4) as it is introduced in [15] and later implemented in [10, 11] for sparse NCD MCs. In particular, we show that one can do better than the method discussed in [15] by intelligently distributing the slack probability mass, $\left(e-P_{\mathcal{A}, \mathcal{A}} e\right)$, among the rows of $P_{\mathcal{A}, \mathcal{A}}$ using the information available in $P_{\mathcal{B}, \mathcal{A}}$. This improved method can be used not only by itself to compute bounds on $\pi_{S_{\mathcal{A}}}$, but also in two-level bounding methods based on decomposition and aggregation to compute bounds on $\pi$. The results in this paper can be combined with reordering of states $[7,10]$ or polynomial transformations [6] to further improve the bounds, and can be extended to continuous-time MCs through uniformization [14].

The next section provides background information on stochastic comparison and the existing method to compute bounds on $\pi_{S_{\mathcal{A}}}$. In section 3 , we develop the improved method and prove that it provides better bounds than the existing method in the strong stochastic sense. Furthermore, we show that there are certain cases in which the bounds are exact. Section 4 includes the results of numerical experiments and in section 5 we conclude.

\section{BACKGROUND ON STOCHASTIC COMPARISON AND THE CURRENT METHOD}

In this section, we present some preliminaries on the stochastic comparison method; the books [9, 13] can be consulted for theoretical issues and different applications of the method. Then we introduce the existing method used to obtain strong stochastic bounds on the conditional steadystate vector of a subset of states in finite, ergodic DTMCs.

\subsection{Strong stochastic order}

We first provide the definition of strong stochastic (st-) comparison over a finite state space. Let $X$ and $Y$ be random variables taking values on the state space $\mathcal{S}=\{1,2, \ldots, n\}$. Let $p$ and $q$ be probability distribution vectors such that

$$
p[j]=\operatorname{Prob}(X=j) \quad \text { and } \quad q[j]=\operatorname{Prob}(Y=j) \quad \forall j \in \mathcal{S} .
$$

Then $X$ is said to be less than $Y$ in the strong stochastic sense, that is $X \leq_{s t} Y$, if and only if

$$
\sum_{j=k}^{n} p[j] \leq \sum_{j=k}^{n} q[j] \quad \forall k \in \mathcal{S} .
$$

Hence, equation (5) defines a partial order on probability distributions, and this order is called the st-order.

Now, we recall the fundamental result which states for two MCs that the st-comparability of their initial probability distributions, the st-monotonicity of one of them, and their st-comparability yield sufficient conditions for their stordering. Let $P$ and $Q$ be DTMCs of order $n$ respectively characterizing the stochastic processes $X(t)$ and $Y(t)$ for $t \in \mathbb{N}$ on $\mathcal{S}$. Then $\{X(t)\}_{t \in \mathbb{N}} \leq_{s t}\{Y(t)\}_{t \in \mathbb{N}}$ (meaning, $X(t) \leq_{s t} Y(t)$ for $\left.\forall t \in \mathbb{N}\right)$ if

(i) $X(0) \leq_{s t} Y(0)$

(ii) st-monotonicity of at least one of the matrices holds; that is, either

$$
P[i, *] \leq_{s t} P[j, *] \quad \forall i, j \in \mathcal{S} \quad \text { such that } i \leq j,
$$

$$
Q[i, *] \leq_{s t} Q[j, *] \quad \forall i, j \in \mathcal{S} \quad \text { such that } i \leq j,
$$

(iii) st-comparability of the matrices holds; that is,

$$
P[i, *] \leq_{s t} Q[i, *] \forall i \in \mathcal{S},
$$

where $P[i, *]$ refers to row $i$ of $P$.

This result has the following implication. If $\{X(t)\}_{t \in \mathbb{N}} \leq_{s t}\{Y(t)\}_{t \in \mathbb{N}}, \lim _{t \rightarrow+\infty} X(t)$ and $\lim _{t \rightarrow+\infty} Y(t)$ exist, and $\pi_{P}$ and $\pi_{Q}$ are respectively the steady-state probability distribution vectors of $P$ and $Q$, then $\pi_{P} \leq_{s t} \pi_{Q}$ (see equation (5)). In other words, $\pi_{Q}\left(\pi_{P}\right)$ provides an st upper (lower)-bound on $\pi_{P}\left(\pi_{Q}\right)$. 


\subsection{Strong stochastic steady-state bounds for a stochastic complement}

As shown in [15], in order to obtain st upper- and lowerbounds on $\pi_{S_{\mathcal{A}}}$, we must first form the DTMCs $\bar{S}_{\mathcal{A}}$ and $\underline{S}_{\mathcal{A}}$ of order $n_{\mathcal{A}}$ such that

$$
\bar{S}_{\mathcal{A}} \leq_{s t} S_{\mathcal{A}} \leq_{s t} \underline{S}_{\mathcal{A}} .
$$

To this end, in Algorithms 1 and 2 we present concise versions of those introduced in [15]. Algorithm 1 places the slack probability mass

$$
\Delta_{\mathcal{A}}=e-P_{\mathcal{A}, \mathcal{A}} e
$$

in the last column of $P_{\mathcal{A}, \mathcal{A}}$ to yield $\bar{S}_{\mathcal{A}}$, whereas Algorithm 2 places it in the first column to yield $\underline{S}_{\mathcal{A}}$. We remark that $\bar{S}_{\mathcal{A}}$ and $S_{\mathcal{A}}$ are minimum and maximum elements of a set of DTMCs bounding $S_{\mathcal{A}}$ respectively from below and above in the strong stochastic sense. However, $\bar{S}_{\mathcal{A}}$ and $S_{\mathcal{A}}$ need not be st-monotone. The time complexity of Algorithms 1 and 2 in the worst-case when $P_{\mathcal{A}, \mathcal{A}}$ is full can be $O\left(n_{\mathcal{A}}^{2}\right)$ floatingpoint arithmetic operations. In their description, $e_{j}$ denotes column $j \in \mathcal{A}$ of $I$.
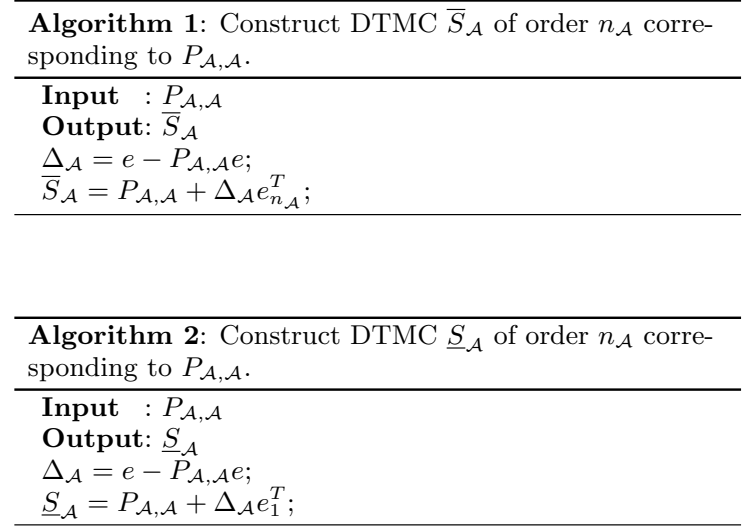

Following Algorithms 1 and 2, the st-monotone upperbounding matrix $\bar{Q}_{\mathcal{A}}$ of order $n_{\mathcal{A}}$ corresponding to $\bar{S}_{\mathcal{A}}$ can be computed by Algorithm 3 and the st-monotone lowerbounding matrix $Q_{\mathcal{A}}$ of order $n_{\mathcal{A}}$ corresponding to $\underline{S}_{\mathcal{A}}$ can be computed by Algorithm 4. Algorithm 3 is given for the first time in [1], whereas Algorithm 4 is the dual of Algorithm 3 for the lower-bounding case and is presented in [10]. The time complexity of their careful implementation in the worst-case when $\bar{S}_{\mathcal{A}}$ and $\underline{S}_{\mathcal{A}}$ are full can be $O\left(n_{\mathcal{A}}^{2}\right)$ floatingpoint arithmetic operations. It is shown in $[10,15]$ that $\bar{Q}_{\mathcal{A}}$ and $\underline{Q}_{\mathcal{A}}$ are st-monotone and

$$
\underline{Q}_{\mathcal{A}} \leq_{s t} S_{\mathcal{A}} \leq_{s t} \bar{Q}_{\mathcal{A}}
$$

implying

$$
\pi_{\underline{Q}_{\mathcal{A}}} \leq_{s t} \pi_{S_{\mathcal{A}}} \leq_{s t} \pi_{\bar{Q}_{\mathcal{A}}}
$$

In the next section, we propose a new method which is based on distributing $\Delta_{\mathcal{A}}$ in equation (6) more intelligently among the columns of $P_{\mathcal{A}, \mathcal{A}}$ and indicate cases in which the bounds may be obtained exactly.
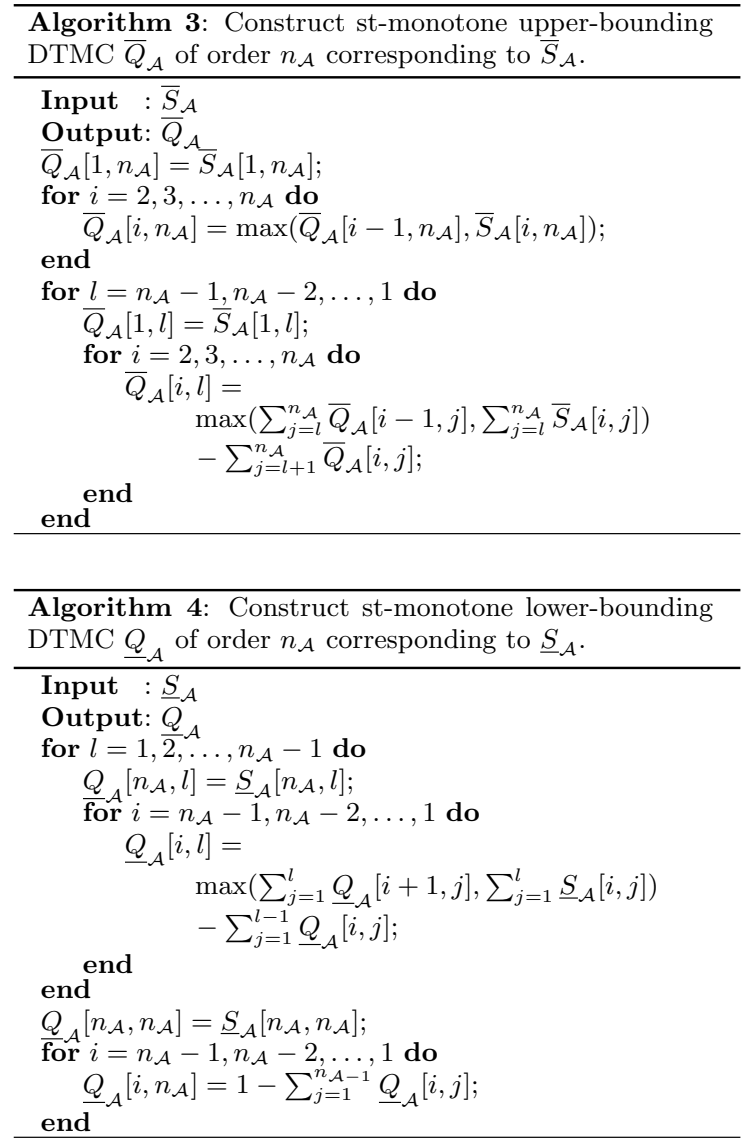

\section{IMPROVING THE STEADY-STATE BOUNDS OF A STOCHASTIC COMPLEMENT}

Our derivation requires us to be able to identify the states within the subsets $\mathcal{A}$ and $\mathcal{B}$ individually and also distinguish between the states of the two subsets symbolically. Hence, in this section we let $\mathcal{A}=\left\{a_{1}, a_{2}, \ldots, a_{n_{\mathcal{A}}}\right\}$ and $\mathcal{B}=\left\{b_{1}, b_{2}, \ldots, b_{n_{\mathcal{B}}}\right\}$.

Now, observe that $\Delta_{\mathcal{A}}\left[a_{i}\right]$ in equation (6) is the total probability of leaving state $a_{i} \in \mathcal{A}$ to go to any state in $\mathcal{B}$, that is,

$$
\begin{aligned}
\Delta_{\mathcal{A}}\left[a_{i}\right] & =1-\sum_{b_{k} \in \mathcal{B}} P_{\mathcal{A}, \mathcal{B}}\left[a_{i}, b_{k}\right] \\
& =\sum_{a_{j} \in \mathcal{A}} H_{\mathcal{A}, \mathcal{A}}\left[a_{i}, a_{j}\right] \\
& =e_{a_{i}}^{T} H_{\mathcal{A}, \mathcal{A}} e \quad \forall a_{i} \in \mathcal{A} .
\end{aligned}
$$

Furthermore, recall from equation (2) that in order to determine the stochastic complement $S_{\mathcal{A}}$, the substochastic matrix $H_{\mathcal{A}, \mathcal{A}}$ must be computed. Indeed, the computation of $H_{\mathcal{A}, \mathcal{A}}$ signifies that we must somehow find a proper way to distribute the slack probability mass $\Delta_{\mathcal{A}}\left[a_{i}\right]$ among the columns $a_{j} \in \mathcal{A}$ by adding to the matrix $P_{\mathcal{A}, \mathcal{A}}$ for all $a_{i} \in \mathcal{A}$. 
Let $B_{b_{k}}\left[a_{i}\right]$ be the probability of leaving $\mathcal{B}$ from state $b_{k} \in \mathcal{B}$ after having entered $\mathcal{B}$, spent some nonnegative time there, left $\mathcal{B}$, and entered $\mathcal{A}$ by state $a_{i} \in \mathcal{A}$. Then the probability of leaving $\mathcal{A}$ by $a_{i} \in \mathcal{A}$ must be equal to the sum of $B_{b_{k}}\left[a_{i}\right]$ for all $b_{k} \in \mathcal{B}$, that is,

$$
\sum_{b_{k} \in \mathcal{B}} B_{b_{k}}\left[a_{i}\right]=\Delta_{\mathcal{A}}\left[a_{i}\right]
$$

Let us denote by $V_{b_{k}}\left[a_{j}\right]$ the probability of entering $\mathcal{A}$ from $\mathcal{B}$ by state $a_{j} \in \mathcal{A}$ given that $\mathcal{B}$ is left from state $b_{k} \in \mathcal{B}$ Then

$$
V_{b_{k}}\left[a_{j}\right]=\frac{P_{\mathcal{B}, \mathcal{A}}\left[b_{k}, a_{j}\right]}{\sum_{a_{l} \in \mathcal{A}} P_{\mathcal{B}, \mathcal{A}}\left[b_{k}, a_{l}\right]} .
$$

As $H_{\mathcal{A}, \mathcal{A}}\left[a_{i}, a_{j}\right]$ represents the probability of leaving $\mathcal{A}$ from state $a_{i} \in \mathcal{A}$ to go to $\mathcal{B}$ and returning to $\mathcal{A}$ by state $a_{j} \in \mathcal{A}$ after having spent some nonnegative time in $\mathcal{B}$, from equation (7) we can write

$$
H_{\mathcal{A}, \mathcal{A}}\left[a_{i}, a_{j}\right]=\sum_{b_{k} \in \mathcal{B}} B_{b_{k}}\left[a_{i}\right] V_{b_{k}}\left[a_{j}\right] .
$$

The fact that $\Delta_{\mathcal{A}}\left[a_{i}\right]$ represents the slack probability mass for state $a_{i} \in \mathcal{A}$ to be stochastic and is equal to $\sum_{a_{j} \in \mathcal{A}} H_{\mathcal{A}, \mathcal{A}}\left[a_{i}, a_{j}\right]$ can be confirmed through equation (9)

$$
\begin{aligned}
\sum_{a_{j} \in \mathcal{A}} H_{\mathcal{A}, \mathcal{A}}\left[a_{i}, a_{j}\right]= & \sum_{a_{j} \in \mathcal{A}} \sum_{b_{k} \in \mathcal{B}} B_{b_{k}}\left[a_{i}\right] V_{b_{k}}\left[a_{j}\right] \\
= & \sum_{b_{k} \in \mathcal{B}} B_{b_{k}}\left[a_{i}\right] \sum_{a_{j} \in \mathcal{A}} V_{b_{k}}\left[a_{j}\right] \\
= & \sum_{b_{k} \in \mathcal{B}} B_{b_{k}}\left[a_{i}\right] \\
& \left(\text { since } \sum_{a_{j} \in \mathcal{A}} V_{b_{k}}\left[a_{j}\right]=1\right) \\
= & \Delta_{\mathcal{A}}\left[a_{i}\right] .
\end{aligned}
$$

\subsection{The case of st upper-bound}

Knowing that $H_{\mathcal{A}, \mathcal{A}}$ is substochastic, for the st upperbounding case, we may try to construct a substochastic ma$\operatorname{trix} F_{\mathcal{A}, \mathcal{A}}$ so that $\bar{S}_{\mathcal{A}}^{\text {new }}=P_{\mathcal{A}, \mathcal{A}}+F_{\mathcal{A}, \mathcal{A}}$ is a DTMC and

$$
\sum_{a_{j}=a_{l}}^{a_{n} \mathcal{A}} H_{\mathcal{A}, \mathcal{A}}\left[a_{i}, a_{j}\right] \leq \sum_{a_{j}=a_{l}}^{a_{n} \mathcal{A}} F_{\mathcal{A}, \mathcal{A}}\left[a_{i}, a_{j}\right] \quad \forall a_{l} \in \mathcal{A}
$$

is satisfied for all $a_{i} \in \mathcal{A}$. If this can be done, then the next result holds.

TheOREm 1. If $F_{\mathcal{A}, \mathcal{A}}$ is defined so that

$$
\bar{S}_{\mathcal{A}}^{\text {new }}=P_{\mathcal{A}, \mathcal{A}}+F_{\mathcal{A}, \mathcal{A}}
$$

is a DTMC and equation (10) is satisfied for all $a_{i} \in \mathcal{A}$, then

$$
S_{\mathcal{A}} \leq_{s t} \bar{S}_{\mathcal{A}}^{\text {new }}
$$

Proof. The result follows from the definition of $S_{\mathcal{A}}$ in equation (2) and the definition of st-comparability in subsection 2.1 of the matrices $S_{\mathcal{A}}$ and $\bar{S}_{\mathcal{A}}^{\text {new }}$ under the given assumptions.
If $F_{\mathcal{A}, \mathcal{A}}=\Delta_{\mathcal{A}} e_{n_{\mathcal{A}}}^{T}$ (i.e., the slack probability mass is placed in the last column as in Algorithm 1), then it is shown in $[10,15]$ that Theorem 1 holds.

The next theorem paves the way to the construction of a substochastic matrix $F_{\mathcal{A}, \mathcal{A}}$ providing more accurate results in the st upper-bounding case.

Theorem 2. If $F_{\mathcal{A}, \mathcal{A}}$ is defined so that

$$
\bar{S}_{\mathcal{A}}^{\text {new }}=P_{\mathcal{A}, \mathcal{A}}+F_{\mathcal{A}, \mathcal{A}}
$$

is a DTMC and for all $a_{i} \in \mathcal{A}$

$$
F_{\mathcal{A}, \mathcal{A}}\left[a_{i}, a_{j}\right]=\left\{\begin{array}{ll}
c_{\mathcal{A}}\left[a_{n_{\mathcal{A}}}\right] \Delta_{\mathcal{A}}\left[a_{i}\right] & a_{j}=a_{n_{\mathcal{A}}} \\
\left(c_{\mathcal{A}}\left[a_{j}\right]-c_{\mathcal{A}}\left[a_{j+1}\right]\right) \Delta_{\mathcal{A}}\left[a_{i}\right] & \text { else }
\end{array},\right.
$$

where

$$
c_{\mathcal{A}}\left[a_{j}\right]=\max _{b_{k} \in \mathcal{B}}\left(\sum_{a_{l}=a_{j}}^{a_{n} \mathcal{A}} V_{b_{k}}\left[a_{l}\right]\right) \quad \forall a_{j} \in \mathcal{A},
$$

then

$$
S_{\mathcal{A}} \leq_{s t} \bar{S}_{\mathcal{A}}^{\text {new }}
$$

Proof. The st-comparison constraints in equation (10) imply that

$$
\sum_{a_{j}=a_{l}}^{a_{n} \mathcal{A}} H_{\mathcal{A}, \mathcal{A}}\left[a_{i}, a_{j}\right] \leq \sum_{a_{j}=a_{l}}^{a_{n} \mathcal{A}} F_{\mathcal{A}, \mathcal{A}}\left[a_{i}, a_{j}\right] \quad \forall a_{l} \in \mathcal{A}
$$

must be satisfied for all $a_{i} \in \mathcal{A}$. To this end, using equation (9) and then equation (7) we first obtain

$$
\begin{aligned}
\sum_{a_{j}=a_{l}}^{a_{n} \mathcal{A}} H_{\mathcal{A}, \mathcal{A}}\left[a_{i}, a_{j}\right] & =\sum_{a_{j}=a_{l}}^{a_{n} \mathcal{A}} \sum_{b_{k} \in \mathcal{B}} B_{b_{k}}\left[a_{i}\right] V_{b_{k}}\left[a_{j}\right] \\
& =\sum_{b_{k} \in \mathcal{B}} B_{b_{k}}\left[a_{i}\right] \sum_{a_{j}=a_{l}}^{a_{n} \mathcal{A}} V_{b_{k}}\left[a_{j}\right] \\
& \leq \sum_{b_{k} \in \mathcal{B}} B_{b_{k}}\left[a_{i}\right] \max _{b_{k} \in \mathcal{B}}\left(\sum_{a_{j}=a_{l}}^{a_{n_{\mathcal{A}}}} V_{b_{k}}\left[a_{j}\right]\right) \\
& \leq \Delta_{\mathcal{A}}\left[a_{i}\right] \max _{b_{k} \in \mathcal{B}}\left(\sum_{a_{j}=a_{l}}^{a_{n} \mathcal{A}} V_{b_{k}}\left[a_{j}\right]\right)
\end{aligned}
$$

for all $a_{i}, a_{l} \in \mathcal{A}$. Next, using the definitions of $F_{\mathcal{A}, \mathcal{A}}\left[a_{i}, a_{j}\right]$ and $c_{\mathcal{A}}\left[a_{j}\right]$ in the statement of the theorem, we obtain

$$
\begin{aligned}
\sum_{a_{j}=a_{l}}^{a_{n}} F_{\mathcal{A}, \mathcal{A}}\left[a_{i}, a_{j}\right]= & \Delta_{\mathcal{A}}\left[a_{i}\right] c_{\mathcal{A}}\left[a_{n_{\mathcal{A}}}\right] \\
& +\Delta_{\mathcal{A}}\left[a_{i}\right] \sum_{a_{j}=a_{l}}^{a_{n_{\mathcal{A}}-1}} c_{\mathcal{A}}\left[a_{j}\right] \\
& -\Delta_{\mathcal{A}}\left[a_{i}\right] \sum_{a_{j}=a_{l}}^{a_{n_{\mathcal{A}}}-1} c_{\mathcal{A}}\left[a_{j+1}\right] \\
= & \Delta_{\mathcal{A}}\left[a_{i}\right] c_{\mathcal{A}}\left[a_{l}\right] \\
= & \Delta_{\mathcal{A}}\left[a_{i}\right] \max _{b_{k} \in \mathcal{B}}\left(\sum_{a_{j}=a_{l}}^{a_{n_{\mathcal{A}}}} V_{b_{k}}\left[a_{j}\right]\right)
\end{aligned}
$$


for all $a_{i}, a_{l} \in \mathcal{A}$, to conclude

$$
\sum_{a_{j}=a_{l}}^{a_{n} \mathcal{A}} H_{\mathcal{A}, \mathcal{A}}\left[a_{i}, a_{j}\right] \leq \sum_{a_{j}=a_{l}}^{a_{n} \mathcal{A}} F_{\mathcal{A}, \mathcal{A}}\left[a_{i}, a_{j}\right] .
$$

Hence, the result is proved.

Using the definition of $V_{b_{k}}\left[a_{j}\right]$ in equation (8), Algorithm 5 constructs the substochastic matrix $F_{\mathcal{A}, \mathcal{A}}$ in Theorem 2. The time complexity of its careful implementation in the worst-case when $P_{\mathcal{A}, \mathcal{A}}$ and $P_{\mathcal{B}, \mathcal{A}}$ are full can be $O\left(n_{\mathcal{A}}\left(n_{\mathcal{A}}+n_{\mathcal{B}}\right)\right)$ floating-point arithmetic operations. In its description, $(x)^{+}=\max (0, x)$.

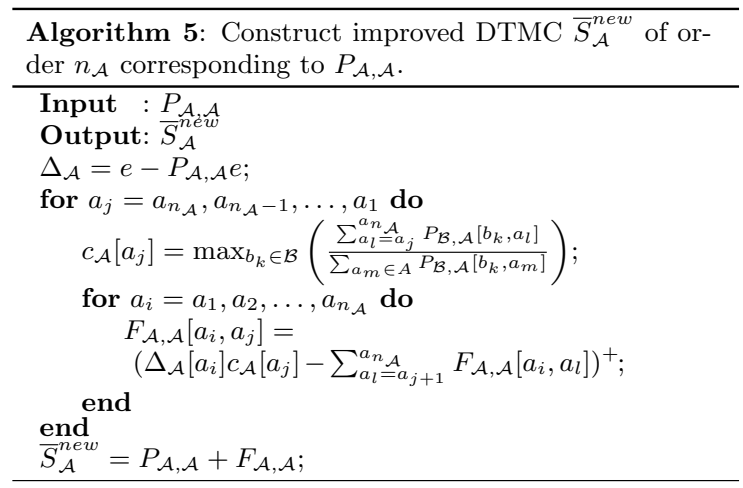

The next lemma shows that the proposed approach is better in the strong stochastic sense than the existing one.

LEMMA 1. If

$$
\bar{S}_{\mathcal{A}}=P_{\mathcal{A}, \mathcal{A}}+\Delta_{\mathcal{A}} e_{n_{\mathcal{A}}}^{T} \quad \text { and } \quad \bar{S}_{\mathcal{A}}^{\text {new }}=P_{\mathcal{A}, \mathcal{A}}+F_{\mathcal{A}, \mathcal{A}},
$$

then

$$
\bar{S}_{\mathcal{A}}^{n e w} \leq_{s t} \bar{S}_{\mathcal{A}}
$$

Proof. Observe that complementing $P_{\mathcal{A}, \mathcal{A}}$ by including the slack probability mass in the last column as in Algorithm 1 corresponds to taking $c_{\mathcal{A}}\left[a_{n_{\mathcal{A}}}\right]=1$ and $c_{\mathcal{A}}\left[a_{j}\right]=0$ for $a_{j} \in \mathcal{A}-\left\{a_{n_{\mathcal{A}}}\right\}$ in Theorem 2 .

\subsection{The case of st lower-bound}

In a similar way to that of the st upper-bounding case, for the st lower-bounding case, we may try to construct a substochastic matrix $G_{\mathcal{A}, \mathcal{A}}$ so that $\underline{S}_{\mathcal{A}}^{\text {new }}=P_{\mathcal{A}, \mathcal{A}}+G_{\mathcal{A}, \mathcal{A}}$ is a DTMC and

$$
\sum_{a_{j}=a_{l}}^{a_{n} \mathcal{A}} G_{\mathcal{A}, \mathcal{A}}\left[a_{i}, a_{j}\right] \leq \sum_{a_{j}=a_{l}}^{a_{n_{\mathcal{A}}}} H_{\mathcal{A}, \mathcal{A}}\left[a_{i}, a_{j}\right] \quad \forall a_{l} \in \mathcal{A}
$$

is satisfied for all $a_{i} \in \mathcal{A}$. For this dual case, we have two theorems and a corresponding lemma, which we present without proofs.

TheOREM 3. If $G_{\mathcal{A}, \mathcal{A}}$ is defined so that

$$
\underline{S}_{\mathcal{A}}^{\text {new }}=P_{\mathcal{A}, \mathcal{A}}+G_{\mathcal{A}, \mathcal{A}}
$$

is a DTMC and equation (11) is satisfied for all $a_{i} \in \mathcal{A}$, then

$$
\underline{S}_{\mathcal{A}}^{n e w} \leq_{s t} S_{\mathcal{A}}
$$

If $G_{\mathcal{A}, \mathcal{A}}=\Delta_{\mathcal{A}} e_{1}^{T}$ (i.e., the slack probability mass is placed in the first column as in Algorithm 2), then it is shown in [10, 15] that Theorem 3 holds.

THEOREM 4. If $G_{\mathcal{A}, \mathcal{A}}$ is defined so that

$$
\underline{S}_{\mathcal{A}}^{\text {new }}=P_{\mathcal{A}, \mathcal{A}}+G_{\mathcal{A}, \mathcal{A}}
$$

is a DTMC and for all $a_{i} \in \mathcal{A}$

$$
G_{\mathcal{A}, \mathcal{A}}\left[a_{i}, a_{j}\right]= \begin{cases}d_{\mathcal{A}}\left[a_{1}\right] \Delta_{\mathcal{A}}\left[a_{i}\right] & a_{j}=a_{1} \\ \left(d_{\mathcal{A}}\left[a_{j+1}\right]-d_{\mathcal{A}}\left[a_{j}\right]\right) \Delta_{\mathcal{A}}\left[a_{i}\right] & \text { else }\end{cases}
$$

where

$$
d_{\mathcal{A}}\left[a_{j}\right]=\max _{b_{k} \in \mathcal{B}}\left(\sum_{a_{l}=a_{1}}^{a_{j}} V_{b_{k}}\left[a_{l}\right]\right) \quad \forall a_{j} \in \mathcal{A},
$$

then

$$
\underline{S}_{\mathcal{A}}^{n e w} \leq_{s t} S_{\mathcal{A}}
$$

Using the definition of $V_{b_{k}}\left[a_{j}\right]$ in equation (8), Algorithm 6 constructs the substochastic matrix $G_{\mathcal{A}, \mathcal{A}}$ in Theorem 4 , whose worst-case time complexity is the same as that of Algorithm 5.

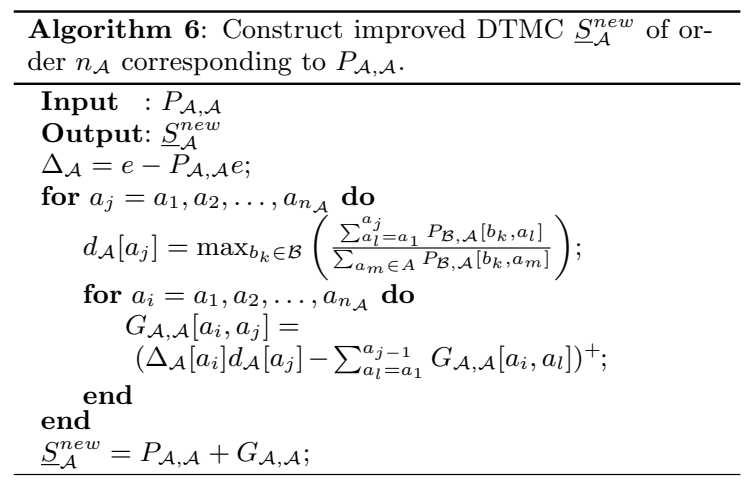

LEMMA 2. If

$$
\underline{S}_{\mathcal{A}}=P_{\mathcal{A}, \mathcal{A}}+\Delta_{\mathcal{A}} e_{1}^{T} \quad \text { and } \quad \underline{S}_{\mathcal{A}}^{\text {new }}=P_{\mathcal{A}, \mathcal{A}}+G_{\mathcal{A}, \mathcal{A}},
$$
then

$$
\underline{S}_{\mathcal{A}} \leq_{s t} \underline{S}_{\mathcal{A}}^{\text {new }} .
$$

\subsection{The cases of exact bounds}

We first state a lemma showing that $H_{\mathcal{A}, \mathcal{A}}$ can be obtained exactly when $P_{\mathcal{B}, \mathcal{A}}$ is a rank-1 matrix.

LEMMA 3. If $P_{\mathcal{B}, \mathcal{A}}=u_{\mathcal{B}} v_{\mathcal{A}}^{T}$, meaning $P_{\mathcal{B}, \mathcal{A}}$ is rank-1, with $v_{\mathcal{A}}^{T} e=1$, then $S_{\mathcal{A}}=P_{\mathcal{A}, \mathcal{A}}+\Delta_{\mathcal{A}} v_{\mathcal{A}}^{T}$.

ProOf. Recall equation (2) and write $H_{\mathcal{A}, \mathcal{A}}$ as in

$$
\begin{aligned}
H_{\mathcal{A}, \mathcal{A}} & =P_{\mathcal{A}, \mathcal{B}}\left(I-P_{\mathcal{B}, \mathcal{B}}\right)^{-1} P_{\mathcal{B}, \mathcal{A}} \\
& =\left(P_{\mathcal{A}, \mathcal{B}}\left(I-P_{\mathcal{B}, \mathcal{B}}\right)^{-1} u_{\mathcal{B}}\right) v_{\mathcal{A}}^{T} \\
& =w_{\mathcal{B}} v_{\mathcal{A}}^{T},
\end{aligned}
$$

where

$$
w_{\mathcal{B}}=P_{\mathcal{A}, \mathcal{B}}\left(I-P_{\mathcal{B}, \mathcal{B}}\right)^{-1} u_{\mathcal{B}}
$$


Now, since $H_{\mathcal{A}, \mathcal{A}} e=e-P_{\mathcal{A}, \mathcal{A}} e=\Delta_{\mathcal{A}}$ from equations (2) and (6), we must have

$$
H_{\mathcal{A}, \mathcal{A}} e=w_{\mathcal{B}}\left(v_{\mathcal{A}}^{T} e\right)=w_{\mathcal{B}}=\Delta_{\mathcal{A}} .
$$

Hence,

$$
H_{\mathcal{A}, \mathcal{A}}=\Delta_{\mathcal{A}} v_{\mathcal{A}}^{T}
$$

and the result is proved.

The next result is based on Lemma 3 and says that the st upper- and lower-bounding DTMCs computed by Algorithms 5 and 6 are equal to the stochastic complement when $P_{\mathcal{B}, \mathcal{A}}$ is a rank-1 matrix.

LeEMma 4. If $P_{\mathcal{B}, \mathcal{A}}=u_{\mathcal{B}} v_{\mathcal{A}}^{T}$ with $v_{\mathcal{A}}^{T} e=1$, then $S_{\mathcal{A}}=$ $\bar{S}_{\mathcal{A}}^{\text {new }}=\underline{S}_{\mathcal{A}}^{\text {new }}$.

Proof. The result follows from Theorems 2 and 4 by observing under the given assumptions that

$$
c_{\mathcal{A}}\left[a_{j}\right]=\sum_{a_{l}=a_{j}}^{a_{n}} v_{\mathcal{A}}\left[a_{l}\right]
$$

and

$$
d_{\mathcal{A}}\left[a_{j}\right]=\sum_{a_{l}=a_{1}}^{a_{j}} v_{\mathcal{A}}\left[a_{l}\right] .
$$

COROLlary 1. When there is a single transition to the subset of interest, Algorithms 5 and 6 yield the stochastic complement.

Proof. If $\mathcal{A}$ is the subset of interest and $P_{\mathcal{B}, \mathcal{A}}$ has a single nonzero, $P_{\mathcal{B}, \mathcal{A}}$ is still a rank-1 matrix.

In the next section, we provide results of numerical experiments on two benchmark problems from the literature and two versions of a small problem.

\section{NUMERICAL EXPERIMENTS}

For brevity, we only present results using Algorithms 1 and 5, and remark that results are reported in four decimal digits after the decimal point; similar results hold for Algorithms 2 and 6.

\subsection{The Courtois problem}

Consider the $(8 \times 8)$ Courtois matrix [4] given by

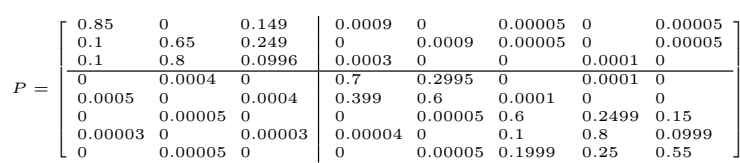

with $\mathcal{A}=\{1,2,3\}, \mathcal{B}=\{4,5,6,7,8\}$, and

$$
\pi=[0.0893,0.0928,0.0405,
$$

$0.1585,0.1189,0.1204,0.2778,0.1018]$.

This is an NCD MC with degree of coupling 0.001 for the chosen partitioning.

The stochastic complement of $P_{\mathcal{A}, \mathcal{A}}$ is given by

$$
S_{\mathcal{A}}=\left[\begin{array}{lll}
0.8503 & 0.0004 & 0.1493 \\
0.1003 & 0.6504 & 0.2493 \\
0.1001 & 0.8002 & 0.0997
\end{array}\right]
$$

with

$$
\pi_{S_{\mathcal{A}}}=[0.4012,0.4168,0.1819] .
$$

The DTMCs computed by Algorithms 1 and 5 are respectively given by

$$
\bar{S}_{\mathcal{A}}=\left[\begin{array}{lll}
0.8500 & 0.0000 & 0.1500 \\
0.1000 & 0.6500 & 0.2500 \\
0.1000 & 0.8000 & 0.1000
\end{array}\right]
$$

and

$$
\bar{S}_{\mathcal{A}}^{\text {new }}=\left[\begin{array}{lll}
0.8500 & 0.0005 & 0.1495 \\
0.1000 & 0.6505 & 0.2495 \\
0.1000 & 0.8002 & 0.0998
\end{array}\right] .
$$

Observe from Algorithm 3 that $\bar{S}_{\mathcal{A}}$ yields the inferior stmonotone upper-bounding DTMC

$$
\bar{Q}_{\mathcal{A}}=\left[\begin{array}{lll}
0.8500 & 0.0000 & 0.1500 \\
0.1000 & 0.6500 & 0.2500 \\
0.1000 & 0.6500 & 0.2500
\end{array}\right]
$$

with

$$
\pi_{\bar{Q}_{\mathcal{A}}}=[0.4000,0.3900,0.2100]
$$

compared to

$$
\bar{Q}_{\mathcal{A}}^{\text {new }}=\left[\begin{array}{lll}
0.8500 & 0.0005 & 0.1495 \\
0.1000 & 0.6505 & 0.2495 \\
0.1000 & 0.6505 & 0.2495
\end{array}\right]
$$

with

$$
\pi_{\bar{Q}_{\mathcal{A}}^{n e w}}=[0.4000,0.3905,0.2095]
$$

given by $\bar{S}_{\mathcal{A}}^{\text {new }}$.

\subsection{The PSW problem}

The second problem that we consider and name $\operatorname{PSW}(\beta)$ comes from the class of $10 \times 10$ matrices used in [12]:

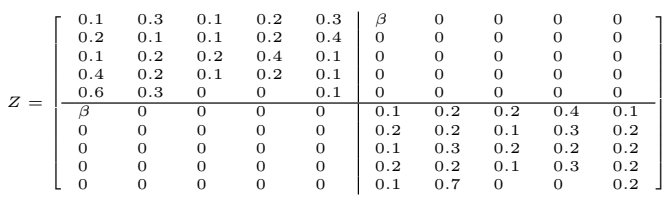

If $D=\operatorname{diag}(1 /(1+\beta), 1,1,1,1,1 /(1+\beta), 1,1,1,1)$, then $P=D Z$ is an NCD MC with degree of coupling $\beta /(1+\beta)$ for the partitioning $\mathcal{A}=\{1,2,3,4,5\}$ and $\mathcal{B}=\{6,7,8,9,10\}$. This problem is interesting also for another reason. The chosen partitioning of states yields one nonzero transition in each of $P_{\mathcal{B}, \mathcal{A}}$ and $P_{\mathcal{A}, \mathcal{B}}$. Here, we consider PSW $\left(10^{-3}\right)$ with the steady-state vector

$$
\begin{aligned}
\pi= & {[0.1009,0.0801,0.0301,0.0603,0.0792,} \\
& 0.1009,0.1967,0.0700,0.1619,0.1198] .
\end{aligned}
$$

The stochastic complement of $P_{\mathcal{A}, \mathcal{A}}$ is given by

$$
S_{\mathcal{A}}=\left[\begin{array}{lllll}
0.1009 & 0.2997 & 0.0999 & 0.1998 & 0.2997 \\
0.2000 & 0.1000 & 0.1000 & 0.2000 & 0.4000 \\
0.1000 & 0.2000 & 0.2000 & 0.4000 & 0.1000 \\
0.4000 & 0.2000 & 0.1000 & 0.2000 & 0.1000 \\
0.6000 & 0.3000 & 0.0000 & 0.0000 & 0.1000
\end{array}\right]
$$


with

$$
\pi_{S_{\mathcal{A}}}=[0.2877,0.2284,0.0860,0.1719,0.2260] .
$$

The DTMCs computed by Algorithms 1 and 5 are respectively given by

$$
\bar{S}_{\mathcal{A}}=\left[\begin{array}{lllll}
0.0999 & 0.2997 & 0.0999 & 0.1998 & 0.3007 \\
0.2000 & 0.1000 & 0.1000 & 0.2000 & 0.4000 \\
0.1000 & 0.2000 & 0.2000 & 0.4000 & 0.1000 \\
0.4000 & 0.2000 & 0.1000 & 0.2000 & 0.1000 \\
0.6000 & 0.3000 & 0.0000 & 0.0000 & 0.1000
\end{array}\right]
$$

and

$$
\bar{S}_{\mathcal{A}}^{\text {new }}=\left[\begin{array}{lllll}
0.1009 & 0.2997 & 0.0999 & 0.1998 & 0.2997 \\
0.2000 & 0.1000 & 0.1000 & 0.2000 & 0.4000 \\
0.1000 & 0.2000 & 0.2000 & 0.4000 & 0.1000 \\
0.4000 & 0.2000 & 0.1000 & 0.2000 & 0.1000 \\
0.6000 & 0.3000 & 0.0000 & 0.0000 & 0.1000
\end{array}\right] .
$$

Observe that $\bar{S}_{\mathcal{A}}^{\text {new }}$ is equal to the stochastic complement. This is not surprising since $P_{\mathcal{B}, \mathcal{A}}$ is of rank-1 with a single nonzero and can be written as $P_{\mathcal{B}, \mathcal{A}}=u_{\mathcal{B}} v_{\mathcal{A}}^{T}$ with $v_{\mathcal{A}}^{T} e=1$, where

$$
u_{\mathcal{B}}^{T}=[0.0010,0,0,0,0] \quad \text { and } \quad v_{\mathcal{A}}^{T}=[1,0,0,0,0]=e_{1}^{T},
$$

Hence, Corollary 1 applies, yielding

$$
S_{\mathcal{A}}=P_{\mathcal{A}, \mathcal{A}}+\Delta_{\mathcal{A}} v_{\mathcal{A}}^{T} .
$$

Now, observe from Algorithm 3 that $\bar{S}_{\mathcal{A}}$ yields the inferior st-monotone upper-bounding DTMC

$$
\bar{Q}_{\mathcal{A}}=\left[\begin{array}{lllll}
0.0999 & 0.2997 & 0.0999 & 0.1998 & 0.3007 \\
0.0999 & 0.2001 & 0.1000 & 0.2000 & 0.4000 \\
0.0999 & 0.2001 & 0.1000 & 0.2000 & 0.4000 \\
0.0999 & 0.2001 & 0.1000 & 0.2000 & 0.4000 \\
0.0999 & 0.2001 & 0.1000 & 0.2000 & 0.4000
\end{array}\right]
$$

with

$$
\pi_{\bar{Q}_{\mathcal{A}}}=[0.0999,0.2101,0.1000,0.2000,0.3901],
$$

which is way off from $\pi_{\bar{S}_{\mathcal{A}}^{n e w}}=\pi_{S_{\mathcal{A}}}$ in the strong stochastic sense.

In passing, we remark that in this problem $P_{\mathcal{A}, \mathcal{B}}$ is also of rank-1. We return to this property in the last problem.

\subsection{Two $5 \times 5$ problems}

In this subsection, we consider two MCs which normally would not be classified as NCD.

\subsubsection{First version}

Consider

$$
P=\left[\begin{array}{lll|ll}
0.1 & 0.2 & 0.4 & 0.2 & 0.1 \\
0.3 & 0.2 & 0 & 0.3 & 0.2 \\
0.1 & 0.3 & 0.2 & 0.1 & 0.3 \\
\hline 0.1 & 0.2 & 0.1 & 0.3 & 0.3 \\
0.2 & 0.4 & 0.2 & 0.1 & 0.1
\end{array}\right]
$$

with $\mathcal{A}=\{1,2,3\}, \mathcal{B}=\{4,5\}$, and

$$
\begin{gathered}
\pi=[0.1713,0.2562,0.1620, \\
0.2105,0.2001] .
\end{gathered}
$$

The stochastic complement of $P_{\mathcal{A}, \mathcal{A}}$ is given by

$$
S_{\mathcal{A}}=\left[\begin{array}{lll}
0.1750 & 0.3500 & 0.4750 \\
0.4250 & 0.4500 & 0.1250 \\
0.2000 & 0.5000 & 0.3000
\end{array}\right]
$$

with

$$
\pi_{S_{\mathcal{A}}}=[0.2905,0.4347,0.2748] .
$$

The DTMCs computed by Algorithms 1 and 5 are respectively given by

$$
\bar{S}_{\mathcal{A}}=\left[\begin{array}{lll}
0.1000 & 0.2000 & 0.7000 \\
0.3000 & 0.2000 & 0.5000 \\
0.1000 & 0.3000 & 0.6000
\end{array}\right]
$$

and

$$
\bar{S}_{\mathcal{A}}^{\text {new }}=S_{\mathcal{A}} .
$$

Observe that $\bar{S}_{\mathcal{A}}^{\text {new }}$ is equal to the stochastic complement. This is not surprising since $P_{\mathcal{B}, \mathcal{A}}$ is of rank-1 and can be written as $P_{\mathcal{B}, \mathcal{A}}=u_{\mathcal{B}} v_{\mathcal{A}}^{T}$ with $v_{\mathcal{A}}^{T} e=1$, where

$$
u_{\mathcal{B}}^{T}=[0.4,0.8] \quad \text { and } \quad v_{\mathcal{A}}^{T}=[0.25,0.5,0.25] .
$$

Hence, Lemma 4 applies, yielding

$$
S_{\mathcal{A}}=P_{\mathcal{A}, \mathcal{A}}+\Delta_{\mathcal{A}} v_{\mathcal{A}}^{T} .
$$

Now, observe from Algorithm 3 that $\bar{S}_{\mathcal{A}}$ yields the inferior st-monotone upper-bounding DTMC

$$
\bar{Q}_{\mathcal{A}}=\left[\begin{array}{lll}
0.1000 & 0.2000 & 0.7000 \\
0.1000 & 0.2000 & 0.7000 \\
0.1000 & 0.2000 & 0.7000
\end{array}\right]
$$

with

$$
\pi_{\bar{Q}_{\mathcal{A}}}=[0.1000,0.2000,0.7000],
$$

which is way off from $\pi_{\bar{S}_{\mathcal{A}}^{\text {new }}}=\pi_{S_{\mathcal{A}}}$ in the strong stochastic sense.

\subsubsection{Second version}

\section{Consider}

$$
P=\left[\begin{array}{lll|ll}
0.1 & 0.2 & 0.4 & 0.2 & 0.1 \\
0.3 & 0.1 & 0 & 0.4 & 0.2 \\
0.1 & 0 & 0 & 0.6 & 0.3 \\
\hline 0.1 & 0.2 & 0 & 0.3 & 0.4 \\
0.2 & 0.4 & 0.2 & 0.1 & 0.1
\end{array}\right]
$$

with $\mathcal{A}=\{1,2,3\}, \mathcal{B}=\{4,5\}$, and

$$
\begin{aligned}
\pi=[0.1637,0.2034,0.1115, \\
\\
0.2914,0.2301] .
\end{aligned}
$$

The stochastic complement of $P_{\mathcal{A}, \mathcal{A}}$ is given by

$$
S_{\mathcal{A}}=\left[\begin{array}{lll}
0.1831 & 0.3661 & 0.4508 \\
0.4661 & 0.4322 & 0.1017 \\
0.3492 & 0.4983 & 0.1525
\end{array}\right]
$$

with

$$
\pi_{S_{\mathcal{A}}}=[0.3420,0.4250,0.2330] .
$$

The DTMCs computed by Algorithms 1 and 5 are respectively given by

$$
\bar{S}_{\mathcal{A}}=\left[\begin{array}{lll}
0.1000 & 0.2000 & 0.7000 \\
0.3000 & 0.1000 & 0.6000 \\
0.1000 & 0.0000 & 0.9000
\end{array}\right]
$$


and

$$
\bar{S}_{\mathcal{A}}^{\text {new }}=\left[\begin{array}{lll}
0.1750 & 0.3500 & 0.4750 \\
0.4500 & 0.4000 & 0.1500 \\
0.3250 & 0.4500 & 0.2250
\end{array}\right] .
$$

Observe from Algorithm 3 that $\bar{S}_{\mathcal{A}}$ yields the inferior stmonotone upper-bounding DTMC

$$
\bar{Q}_{\mathcal{A}}=\left[\begin{array}{lll}
0.1000 & 0.2000 & 0.7000 \\
0.1000 & 0.2000 & 0.7000 \\
0.1000 & 0.0000 & 0.9000
\end{array}\right]
$$

with

$$
\pi_{\bar{Q}_{\mathcal{A}}}=[0.1000,0.0250,0.8750]
$$

compared to

$$
\bar{Q}_{\mathcal{A}}^{\text {new }}=\left[\begin{array}{lll}
0.1750 & 0.3500 & 0.4750 \\
0.1750 & 0.3500 & 0.4750 \\
0.1750 & 0.3500 & 0.4750
\end{array}\right]
$$

with

$$
\pi_{\bar{Q}_{\mathcal{A}}^{\text {new }}}=[0.1750,0.3500,0.4750]
$$

given by $\bar{S}_{\mathcal{A}}^{\text {new }}$

We remark that although $P_{\mathcal{A}, \mathcal{B}}$ is a rank-1 matrix, $\bar{S}_{\mathcal{A}}^{\text {new }} \neq$ $S_{\mathcal{A}}$. Hence, a result similar to Lemma 4 does not hold for the case of a rank-1 $P_{\mathcal{A}, \mathcal{B}}$, and this problem serves as the counter-example.

\section{CONCLUSION}

In this contribution, we have given algorithms that construct st upper- and lower-bounding DTMCs on a submatrix associated with a subset of states in a finite, irreducible, and aperiodic DTMC. These DTMCs have been shown to provide better bounds in the strong stochastic sense than DTMCs constructed with the existing approach, and are therefore recommended in bounding the conditional steadystate probability distribution vector of the subset of states. In particular, the results with the proposed approach are shown to be exact when the submatrix representing the transitions from states outside the subset of interest to the states in the subset of interest is of rank-1.

Although we have concentrated on bounding the conditional steady-state vector of a subset of states in finite, ergodic DTMCs, the results in this paper can be extended to bounding the conditional transient probability distribution of the subset of interest.

\section{REFERENCES}

[1] O. Abu-Amsha and J.-M. Vincent. An algorithm to bound functionals of Markov chains with large state space. In 4th INFORMS Conference on Telecommunications, Boca Raton, Florida, 8-11 March 1998. Available as Rapport de recherche MAI No. 25, IMAG, Grenoble, France, 1996.

[2] A. Berman and R. J. Plemmons. Nonnegative matrices in the Mathematical Sciences. SIAM Press,
Philadelphia, Pennsylvania, 1994.

[3] D. A. Bini, G. Latouche, and B. Meini. Numerical Methods for Structured Markov Chains. Oxford University Press, Oxford, England, 2005.

[4] P.-J. Courtois. Decomposability: Queueing and Computer System Applications. Academic Press, New York, 1977.

[5] P.-J. Courtois and P. Semal. Bounds for the positive eigenvectors of nonnegative matrices and for their approximations by decomposition. Journal of the Association for Computing Machinery, 31:804-825, 1984.

[6] T. Dayar, J.-M. Fourneau, N. Pekergin, and J.-M Vincent. Polynomials of a stochastic matrix and strong stochastic bounds. In MAM 2006: Markov Anniversary Meeting, Eds. A. N. Langville and W. J. Stewart, Boson Books, Raleigh, North Carolina, 211-228, 2006

[7] T. Dayar and N. Pekergin. Stochastic comparison, reorderings, and nearly completely decomposable Markov chains. In Numerical Solution of Markov Chains, Eds, W. J. Stewart, B. Plateau, and M. Silva, Prensas Universitarias de Zaragoza, Zaragoza, Spain, 228-246, 1999

[8] C. D. Meyer. Stochastic complementation, uncoupling Markov chains and the theory of nearly reducible systems. SIAM Review, 31(2):240-272, 1989.

[9] A. Muller and D. Stoyan. Comparison Methods for Stochastic Models and Risks. Wiley, New York, 2002.

[10] N. Pekergin, T. Dayar, and D. N. Alparslan, Componentwise bounds for nearly completely decomposable Markov chains using stochastic comparison and reordering. Technical Report BU-CE-0202, Department of Computer Engineering, Bilkent University, Ankara, Turkey, 2002. Available at http://www.cs.bilkent.edu.tr/ tech-reports/2002/BU-CE-0202.ps.gz Last accessed on May 20, 2006.

[11] N. Pekergin, T. Dayar, and D. N. Alparslan. Componentwise bounds for nearly completely decomposable Markov chains using stochastic comparison and reordering. European Journal of Operational Research, 165:810-825, 2005.

[12] C. C. Paige, P. H. Styan, and P. G. Wachter. Computation of the stationary distribution of a Markov chain. Journal of Statististical Computation and Simulation, 4:173-186, 1975.

[13] M. Shaked and J. G. Shantikumar. Stochastic Orders and Their Applications. Academic Press, San Diego, California, 1994

[14] W. J. Stewart. Introduction to the Numerical Solution of Markov Chains. Princeton University Press, Princeton, New Jersey, 1994

[15] L. Truffet. Near complete decomposability: bounding the error by stochastic comparison method. Advances in Applied Probability, 29:830-855, 1997. 